SURVEY PAPER

\author{
Enrique Llorens-Fuster
}

\title{
The fixed point property for renormings of $\ell_{2}$
}

Received: 1 August 2012 / Accepted: 7 September 2012 / Published online: 7 November 2012

(C) The Author(s) 2012. This article is published with open access at Springerlink.com

\begin{abstract}
A famous open question in metric Fixed Point Theory is whether every Banach space which is isomorphic to the Hilbert space $\ell_{2}$ has the fixed point property for nonexpansive mappings. We give an overview about the state of the advances towards their solution.
\end{abstract}

Mathematics Subject Classification 47H10 - 46B20

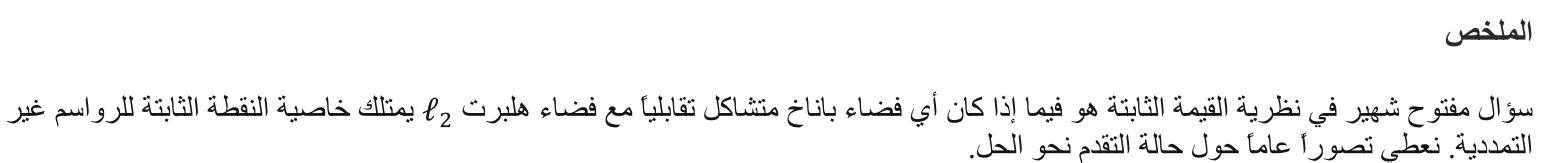

\section{Introduction}

Let $C$ be a subset of a Banach space $(X,\|\cdot\|)$. A mapping $T: C \rightarrow X$ is called nonexpansive whenever $\|T(x)-T(y)\| \leq\|x-y\|$ for all $x, y \in C$. For example, isometries, contractions and resolvents of accretive mappings are all nonexpansive.

The Fixed Point Theory for nonexpansive mappings is rich and varied. It represents a border case between the Banach contraction mapping principle and Schauder's theorem, and moreover it is significant because of its close connections with the theory of accretive operators, variational inequalities and hence optimization and nonlinear analysis in general.

The Banach space $(X,\|\cdot\|)$ has the fixed point property (FPP) if every nonexpansive self-mapping of each nonempty bounded closed convex subset $C$ of $X$ has a fixed point. If the same property holds for every weakly compact convex subset of $X$ we say that $(X,\|\cdot\|)$ has the weak fixed point property (WFPP for short). For instance, the classical Banach space $c_{0}$ enjoys the WFPP but it lacks the FPP.

After a seminal result due to Kirk [39] it was known that both the FPP and the WFPP strongly depend on 'nice' geometrical properties of the space under consideration. More precisely, Kirk's result establishes that those Banach spaces with normal structure (NS) have the WFPP. In particular uniformly convex Banach spaces, and hence Hilbert spaces, have normal structure.

For some time was unknown if every Banach space could have the WFPP. However, Alspach [1] showed that $L^{1}([0,1])$ fails this property. In 1997 Lennard and Dowling (see [11]) proved that a subspace of $L^{1}([0,1])$ has the WFPP if and only if it is reflexive. This fact supported the conjecture that a general Banach space could enjoy FPP if and only if it is reflexive. But P.K. Lin in 2008 showed that the classical (nonreflexive) space $\ell_{1}$ can be equivalently renormed to fail the FPP. However a long-time open question in Metric Fixed Point Theory

E. Llorens-Fuster $(\varangle)$

Dept. Análisis Matemático, Facultad de Matemáticas, Burjassot 46100, Valencia, Spain

E-mail: enrique.llorens@uv.es 
remains open: Does every reflexive Banach space have the FPP? (see [40] for more about this problem). A particular case of this question is: Does every superreflexive Banach space have the FPP? Although superreflexive spaces have the FPP for isometries [45], the question for general nonexpansive mappings remains unsolved in spite of many investigations in this direction.

A major complication in the general study of the FPP is the fact that FPPs are not invariant under renormings, that is, the FPP is not a topological but metric property. In fact, it is not known whether there exists a nontrivial class of isomorphic Banach spaces such that every member of this class has the FPP. [A trivial class with this property are the finite dimensional Banach spaces. In the same way, every Banach space isomorphic to a Schur space enjoys the WFPP.]

Since Hilbert spaces are superreflexive and have normal structure, and hence they enjoy the FPP, a natural question arises. Does any Banach space which is isomorphic to a Hilbert space enjoy the FPP? This problem is essentially of separable naturae [27, p. 35]. Thus, without loss of generality we can merely ask:

Does every (equivalent) renorming of $\ell_{2}$ have the FPP?

In these notes we will be mainly concerned with an updated survey on what is known about the partial answers to Problem [P]. In particular we will devote Sect. 3 to overview the most well-known geometrical properties which imply the FPP in the framework of the Banach spaces $X=\left(\ell_{2},\|\cdot\|\right)$ where $\|\cdot\|$ stands for a norm on $\ell_{2}$ equivalent to the standard one. In Sect. 5, we will give a renorming of $\ell_{2}$ lacking of many of them, which still has the FPP. Most of the results presented here are taken from [33,34] and [16].

\section{Notations and basic definitions}

All the results of this paper are established in $\ell_{2}$, the classical real space of all sequences $x=\left(x_{n}\right)=(x(n))$ for which $\sum_{i=1}^{\infty} x_{i}^{2}<\infty$. The Euclidean norm $\|x\|_{2}:=\sqrt{\sum_{i=1}^{\infty} x_{i}^{2}}$ is associated to the ordinary inner product $\langle x, y\rangle=\sum_{n=1}^{\infty} x_{n} y_{n}$. Also the "sup" norm $\|x\|_{\infty}=\sup \{|x(n)|: n=1, \ldots\}$ will be sometimes considered. The standard Schauder basis of $\left(\ell_{2},\|\cdot\|_{2}\right)$ will be denoted by $\left(e_{n}\right)$, and the weak convergence of a sequence $\left(x_{n}\right)$ in $\ell_{2}$ to $x_{0} \in X$ as $x_{n} \rightarrow x_{0}$.

If $\|$.$\| is a norm on \ell_{2}$ equivalent to $\|.\|_{2}$, we merely will say that $\|$.$\| is a renorming of \ell_{2}$. Given such a renorming, we will denote the closed balls and the spheres as follows: $B_{\|.\|}:=\left\{x \in \ell_{2}:\|x\| \leq 1\right\}$, $S_{\|.\|}:=\left\{x \in \ell_{2}:\|x\|=1\right\}$.

Recall that the modulus of convexity of $(X,\|\cdot\|)$ is the function $\delta_{X}:[0,2] \rightarrow[0,1]$ given by

$$
\delta_{X}(\varepsilon):=\inf \left\{1-\left\|\frac{1}{2}(x+y)\right\|: x, y \in B_{X},\|x-y\| \geq \varepsilon\right\} .
$$

The characteristic of convexity of $(X,\|\cdot\|)$ is the real number

$$
\varepsilon_{0}(X):=\sup \left\{\varepsilon \in[0,2]: \delta_{X}(\varepsilon)=0\right\} .
$$

The space $(X,\|\cdot\|)$ is uniformly convex whenever $\varepsilon_{0}(X)=0$. For a bounded sequence $\left(x_{n}\right)$ in $X$ we will use the notation

$$
D\left(x_{n}\right):=\underset{n}{\limsup }\left(\limsup _{m}\left\|x_{n}-x_{m}\right\|\right) .
$$

\section{The state of art}

From 1965 until now a considerable amount of papers dealing with geometrical properties of the Banach spaces which are sufficient conditions for WFPP have been published. Among others one can list the following:

(1) Normal structure (Kirk [39]).

(2) Asymptotic normal structure (Baillon and Schöneberg [4]).

(3) Orthogonal convexity (Jiménez-Melado [29]). 
(4) Uniform noncreasyness (Prus [52]) and subsequent generalizations due to several authors, as for instance, Fetter, Gamboa de Buen, García-Falset, and others (see [13,15,23]).

(5) Condition $R(X)>2$ (García-Falset [20]).

(6) Condition $M(X)>1$ (Domínguez-Benavides [9]).

(7) Uniform nonsquareness $\left(\varepsilon_{0}(X)<2\right)$ (Mazcuñán [24,50]).

(8) Prus-Sczepanik condition [53].

(9) E-convexity (Dowling et al. [12]).

(10) Property WORTH along with reflexivity (Fetter and Gamboa [14]).

Moreover, several sufficient conditions for WFPP are not properly geometrical properties. For instance, in the framework of the (equivalent) renormings of $\ell_{2}$ are, among others:

(1) to have a Schauder basis nice enough (Lin, Khamsi et al., around 1985),

(2) $d\left(X, X_{H}\right)<\sqrt{\frac{5+\sqrt{17}}{2}}$, where $X_{H}$ any Hilbert renorming of $\ell_{2}$, (Mazcuñán [50]).

Some other sufficient conditions for WFPP are stated in terms of geometrical properties of more sophisticated structures, as ultrapowers of the space under consideration. Due to the difficulty of checking these properties in concrete spaces, we will not consider them here. For instance one can mention the following:

(1) The property AMC introduced in 1993 (see [21]).

(2) The Wiśnicki results ensuring the FPP for any superreflexive Banach space, say $X$, such that it admits an suitable ultrapower $(X)_{\mathcal{U}}$ enjoying the so called property $\left(S_{m}\right)$ (see [66]).

The relationships between most of the above properties have remained hidden for years. Maybe because to check that a given Banach space enjoys one of these conditions is not an easy task. For instance, whether the uniformly non-square Banach spaces (i.e. spaces $X$ for which $\varepsilon_{0}(X)<2$ ) have FPP was a long-time open question. In 2003 it was discovered (see $[24,50])$ that all these spaces indeed satisfy the above-mentioned Dominguez Benavides' condition $M(X)>1$ (see [9]).

In the same way, a question posed by Sims [55], namely if (reflexive) spaces with the WORTH property have FPP was solved by Fetter and Gamboa [14], realizing that such spaces in fact enjoy the AMC property, defined by García-Falset [17].

In this section we will try to clarify and separate these sufficient conditions, focusing our overview on a seemingly unknown implication between any two of them.

\subsection{Normal structure and its generalization}

Without doubt, the most relevant sufficient condition for the FPP in the Banach spaces is the normal structure. Recall that a Banach space $(X,\|\cdot\|)$ is said to have normal structure if for each nontrivial bounded convex subset $C$ of $X$ there exists a nondiametral point $p \in C$, that is a point $p \in C$ such that

$$
\sup \{\|p-x\|: x \in C\}<\operatorname{diam}(C) .
$$

This property was introduced by Brodskii and Milman. As we pointed out above, the seminal Kirk's result of 1965 was to show that reflexive spaces with normal structure have FPP [39]. This work was the starting point of considerable research aimed at finding other geometrical properties of Banach spaces which imply normal structure. Among many others one can list the following (see [27,58] for references and more information):

(1) Uniform convexity (UC) (Belluce [5]).

(2) $\varepsilon_{0}(X)<1$ (Goebel [26]).

(3) Uniform convexity in every direction (Zidler [67]).

(4) Opial condition (Gossez Lami Dozo [28]).

(5) $k$-uniform convexity (Sullivan [61]).

(6) $\operatorname{WCS}(X)>1[8]$.

(7) Near uniform convexity (Van Dulst [64]).

(8) Uniform smoothness (Turret [63]).

(9) Property weak uniform Kadek-Klee (Van Dulst and Sims [65]).

(10) Property P (Tan and Xu [62]).

(11) Property GGLD (Jiménez Melado [30]).

(12) Property Asymptotic P (Sims and Smyth [57]). 
Notice that one can find "aberrant" renormings of $\ell_{2}$ with normal structure which lack uniform convexity and several of its generalizations (see [25,59]).

Example 3.1 Let $E_{\beta}$ be $\ell_{2}$ endowed with the norm $|x|_{\beta}:=\max \left\{\|x\|_{2}, \beta\|x\|_{\infty}\right\}$.

Karlovitz [36] first noted that, even in reflexive spaces, normal structure is not essential for FPP. In fact, he realized that $E_{\sqrt{2}}$ fails to have normal structure and it enjoys FPP.

The concept of asymptotic normal structure (ANS) was introduced by Baillon and Schöneberg in 1981 (see [4]).

Definition 3.2 A Banach space $(X,\|\cdot\|)$ has asymptotic normal structure if each nonempty bounded closed and convex subset $C$ of $X$ which contains more than one point has the property: If a sequence $\left(x_{n}\right)$ in $C$ satisfies $\left\|x_{n}-x_{n+1}\right\| \rightarrow 0$, there exists a point $x \in C$ such that

$$
\lim \inf \left\|x-x_{n}\right\|<\operatorname{diam}(\mathrm{C}) .
$$

Clearly, if $(X,\|\cdot\|)$ has NS then it has ANS but the converse is not true. For example, for $\beta \in[\sqrt{2}, 2)$ the spaces $E_{\beta}$ have ANS but they lack of NS. In the same work [4] the following result was shown.

Theorem 3.3 Every reflexive Banach space with asymptotic normal structure has the FPP.

There are a wide literature about further features of normal structure. For instance one can see the comprehensive articles $[49,58]$ as well as the books $[3,27,38,42]$ and the references therein.

\subsection{Orthogonal convexity}

Some sufficient conditions for the WFPP are independent of the normal structure. One of them is the so called orthogonal convexity, a property of geometric nature which, among other things, implies the Banach-Saks property (see [41]). It is known that every uniformly convex Banach space is orthogonally convex. Other examples include $E_{\beta}, \ell_{1}, c_{0}, c$ and the standard nonreflexive James space $J$. To give the precise definition of orthogonal convexity, we need some further notation. Let $(X,\|\cdot\|)$ a normed space. If $x, y \in X$,

$$
M_{\beta}(x, y):=\left\{z \in X: \max \{\|z-x\|,\|z-y\|\} \leq \frac{1}{2}(1+\beta)\|x-y\|\right\}
$$

and

$$
A_{\beta}\left[\left(x_{n}\right)\right]:=\limsup _{n}\left(\limsup _{m}\left|M_{\beta}\left(x_{n}, x_{m}\right)\right|\right),
$$

where $|A|:=\sup \{\|v\|: v \in A\}$ for any nonempty bounded subset $A$ of $X$.

Definition 3.4 A Banach space $(X,\|\|$.$) is orthogonally convex [(\mathrm{OC})$ for short] if for every weakly null sequence $\left(x_{n}\right)$ with $D\left[\left(x_{n}\right)\right]>0$ there exists $\beta>0$ such that $A_{\beta}\left[\left(x_{n}\right)\right]<D\left[\left(x_{n}\right)\right]$.

Example 3.5 Let us consider the following renorming of $\ell_{2}$. For $x \in \ell_{2}$

$$
\|x\|:=\max \left\{\frac{1}{3}\|x\|_{2}, \sup _{n \geq 2}|x(1)+x(n)+x(n+1)|\right\}
$$

This norm was introduced by van Dulst [64]. The space $V D:=\left(\ell_{2},\|\cdot\|\right)$ lacks ANS, although it is orthogonally convex (see [31]).

The main result in [29] and [31] is the following.

Theorem 3.6 Every orthogonally convex Banach space has the WFPP.

Hence reflexive OC Banach spaces have FPP.

Further developments and generalizations on the notion of orthogonal convexity can be found in [7,21,62], and [41] among others.

On the other hand, there are some renormings of $\ell_{2}$ with normal structure which fail to be OC. The following example can be found in [29]. 
Example 3.7 For $x \in \ell_{2}$ let

$$
|x|:=\max \left\{\|x\|_{2}, \sup \{|x(k)|+|x(r)|: 1 \leq k<r\}\right\}+\sum_{n=1}^{\infty} \frac{|x(n)|}{2^{n}} .
$$

In fact, in [29] it was shown that $Z:=\left(\ell_{2},||.\right)$ satisfies the so called Opial condition, which in turn implies that $Z$ has normal structure.

Nevertheless, the sequence $\left(e_{n}\right)$ is weakly null. If $m \neq n$,

$$
\left|e_{m}-e_{n}\right|=2+2^{-m}+2^{-n}
$$

which implies that $D\left(e_{n}\right)=2$.

Given $\lambda>0$ there exist positive integers $p, q$ such that for $n \geq p$ and $m \geq q$,

$$
\left.\begin{array}{l}
1+2^{-n} \leq \frac{1}{2}(1+\lambda)\left(2+2^{-n}+2^{-m}\right) \\
1+2^{-m} \leq \frac{1}{2}(1+\lambda)\left(2+2^{-n}+2^{-m}\right)
\end{array}\right\}
$$

For $m \neq n$ take $z:=z(m, n):=e_{n}+e_{m}$. Since

$$
\left.\begin{array}{l}
\left|z-e_{n}\right|=\left|e_{m}\right|=1+2^{-m} \\
\left|z-e_{m}\right|=\left|e_{n}\right|=1+2^{-n}
\end{array}\right\}
$$

we have $z \in M_{\lambda}\left(e_{n}, e_{m}\right)$. Moreover $|z|=2+2^{-m}+2^{-n}$, thus

$$
\left|M_{\lambda}\left(e_{m}, e_{n}\right)\right| \geq 2=D\left(e_{n}\right)
$$

which in turn implies that $A_{\lambda}\left(e_{n}\right)=D\left(e_{n}\right)$ and hence $\left(\ell_{2},|\cdot|\right)$ is not OC.

\subsection{From (UNC) Banach spaces to Prus-Szczepanik condition}

Recall that a Banach space $(X,\|\cdot\|)$ is weakly nearly uniformly smooth (WNUS) if for some $\varepsilon>0$ there exists $\mu>0$ such that if $0<t<\mu$ and $\left(x_{n}\right)$ is a basic sequence in $B_{X}$ then there exists $k>1$ so that $\left\|x_{1}+t x_{k}\right\| \leq 1+\varepsilon t$.

A coefficient closely related with this property was defined by García-Falset in 1994 (see [18]), as follows:

$$
R(X):=\sup \left\{\liminf _{n \rightarrow \infty}\left\|x_{n}+x\right\|: x, x_{n} \in B_{X}(n=1,2, \ldots), x_{n} \rightarrow 0_{X}\right\} .
$$

For example, $R\left(c_{0}\right)=1$ and $R\left(\left(\ell_{2},\|\cdot\|_{2}\right)\right)=\sqrt{2}$. It turns out that a Banach space $(X,\|\cdot\|)$ is WNUS if and only if it is reflexive and $R(X)<2$. In 1997 (see [20]) the following result was proven.

Theorem 3.8 If $R(X)<2$ then $(X,\|\cdot\|)$ has WFPP.

Example 3.9 Let $V=\left(\ell_{2},\|\cdot\|\right)$ be the above-mentioned van Dulst space. Since $-e_{n} \in B_{V}(n=1,2, \ldots)$ and $-e_{n} \rightarrow 0_{\ell_{2}}$, then

$$
R(V) \geq \liminf _{n \rightarrow \infty}\left\|-e_{n}+e_{1}\right\|=2 .
$$

Thus, $V$ is orthogonally convex but it is not WNUS.

Condition $R(X)<2$ is, among those implying WFPP, one of the easiest to check. However, it was weakened by Domínguez-Benavides (see [9]) as follows. For $a \geq 0$, and a Banach space $(X,\|\cdot\|)$, put

$$
R(a, X):=\sup \left\{\liminf \left\|x+x_{n}\right\|\right\},
$$

where the supremum is taken over all $x \in X$ with $\|x\| \leq a$ and all weakly null sequences $\left(x_{n}\right)$ in the unit ball of $X$ such that $D\left(x_{n}\right) \leq 1$. Next, define

$$
M(X):=\sup \left\{\frac{1+a}{R(a, X)}: a \geq 0\right\} .
$$

The class of the Banach spaces $X$ with $M(X)>1$ properly contains those Banach spaces with $R(X)<2$. The main result in [9] is the following. 
Theorem 3.10 If $M(X)>1$, then $X$ has the WFPP.

This last result was very important in the development of this part of the theory because Eva Mazcuñan Navarro showed in 2003 (see [24,50]) the following result, solving a long-time open question.

Theorem 3.11 Every Banach space $X$ with characteristic of convexity $\varepsilon_{0}(X)<2$ satisfies the condition $M(X)>1$ and hence has the FPP.

Remark 3.12 In summary, if $X$ is a reflexive Banach space, the following implications hold.

$$
\begin{gathered}
R(X)<2 \\
\varepsilon_{0}(X)<2 \Rightarrow M(X)>1 \Rightarrow \text { FPP }
\end{gathered}
$$

Thus, if there exists a renorming, say $\|\cdot\|$, of $\ell_{2}$ lacking FPP, then $M\left(\left(\ell_{2},\|\cdot\|\right)\right)=1$ and $\varepsilon_{0}\left(\left(\ell_{2},\|\cdot\|\right)\right)=2$.

A celebrated sufficient condition for WFPP was introduced in 1997 by Prus (see [52]). Given two functionals $x^{*}, y^{*} \in S_{X^{*}}$, and a scalar $\delta \in[0,1]$, we put

$$
S\left(x^{*}, \delta\right):=\left\{x \in B_{X}: x^{*}(x) \geq 1-\delta\right\}
$$

and

$$
S\left(x^{*}, y^{*}, \delta\right):=S\left(x^{*}, \delta\right) \cap S\left(y^{*}, \delta\right) .
$$

The following definitions were also given in [52]: that

We say that the unit sphere $S_{X}$ of a Banach space $X$ has a crease if there are $x^{*}, y^{*} \in S_{X^{*}}, x^{*} \neq y^{*}$, such

$$
\operatorname{diam}\left(S\left(x^{*}, y^{*}, 0\right)\right)>0 .
$$

A Banach space $X$ is called noncreasy if $S_{X}$ does not have a crease.

We say that a Banach space $X$ is uniformly noncreasy (UNC in short) if for every $\varepsilon>0$ there exists $\delta>0$ such that, for any $x^{*}, y^{*} \in S_{X^{*}}$ with $\left\|x^{*}-y^{*}\right\| \geq \varepsilon$, we have

$$
\operatorname{diam} S\left(x^{*}, y^{*}, \delta\right) \leq \varepsilon .
$$

Examples of UNC Banach spaces are the uniformly convex and uniformly smooth Banach spaces. The following is the main result of [52].

Theorem 3.13 UNC Banach spaces have WFPP.

In particular, the space $E_{\sqrt{2}}$ is UNC but does not have NS. There are UNC renormings of $\ell_{2}$ but failing to be both OC and NUS. Uniform noncreasyness is a self dual property.

Closely inspired by Prus' paper [52], several classes of Banach spaces more general than UNC and still enjoying FPP were later defined. For instance.

(1) In [23] the $r$-uniformly noncreasy Banach spaces.

(2) In [15] the $(r, k)$-somewhat uniformly noncreasy ( $r$-SUNC) Banach spaces that properly contain the $r$-SUNC spaces.

(3) In [13] the $(r, k, l)$-somewhat uniformly noncreasy Banach spaces that properly contain the $(r, k)$-SUNC spaces.

In 2005, Prus and Szczepanik introduced in [53] a class of spaces which they called nearly uniformly noncreasy (NUNC). In their definition they combined the concepts of infinite-dimensional uniform convexity and uniform smoothness. This class contains the UNC spaces as well as the spaces considered in $[13,15,23]$ and all the nearly uniformly convex spaces, among others.

Assume now that a Banach space $X$ lacks the Schur property. Then the family $\mathfrak{N}_{X}$ of all weakly null sequences $\left(x_{n}\right)$ in $S_{X}$ is nonempty. Prus and Szczepanik defined the following moduli.

Given $\varepsilon \geq 0$ and $x \in X$, put

$$
d(\varepsilon, x):=\inf _{\left(y_{m}\right) \in \mathfrak{N}_{X}} \limsup _{m \rightarrow \infty}\left\|x+\varepsilon y_{m}\right\|-\|x\|
$$


and

$$
b(\varepsilon, x)=\sup _{\left(y_{m}\right) \in \mathfrak{N}_{X}} \liminf _{m \rightarrow \infty}\left\|x+\varepsilon y_{m}\right\|-\|x\| .
$$

By $\mathfrak{M}_{X}$ we denote the set of all weakly null sequences $\left(y_{n}\right)$ in $B_{X}$ such that $D\left(y_{n}\right) \leq 1$. Let $x \in X$ and $\varepsilon \geq 0$. We put

$$
b_{1}(\varepsilon, x):=\sup _{\left(y_{m}\right) \in \mathfrak{M}_{X}} \liminf _{m \rightarrow \infty}\left\|x+\varepsilon y_{m}\right\|-\|x\| .
$$

Observe that "lim inf" can be replaced by "lim sup" in the definition of $b_{1}(\varepsilon, x)$. It follows that $b_{1}(\epsilon, x)$ is a convex function of $\varepsilon \in[0,+\infty)$. Moreover, $b_{1}(0, x)=0$, so $b_{1}(\varepsilon, x) / \varepsilon$ is nondecreasing in the interval $(0,+\infty)$.

A Banach space is said to be NUNC if either it is a Schur space or for every $\varepsilon>0$ there exists $t>0$ such that for every $x \in S_{X}$ it is the case that $d(\varepsilon, x) \geq t$ or $b(t, x) \leq \varepsilon t$.

Every UNC Banach space is NUNC and the converse does not hold. The NUNC spaces contains, among others, all nearly uniformly convex and all nearly uniformly smooth spaces. A reflexive Banach space is NUNC if and only if its dual is NUNC.

The main fixed point theorem in [53] reads as follows.

Theorem 3.14 Let $X$ be a Banach space without the Schur property. If there exists $\varepsilon \in(0,1)$ such that for every $x \in S_{X}$ it is the case that $b_{1}(1, x)<1-\varepsilon$ or $d(1, x)>\varepsilon$, then $X$ has the weak fixed point property.

For the sake of clarity it is convenient to consider the class of all Banach spaces satisfying the assumptions of the above theorem. Notice that this class contains, in particular, all the NUNC Banach spaces.

Definition 3.15 Let $(X,\|\cdot\|)$ be a non-Schur Banach space. If there exists $\varepsilon \in(0,1)$ such that for every $x \in S_{X}$ it is the case that $b_{1}(1, x)<1-\varepsilon$ or $d(1, x)>\varepsilon$ we say that $(X,\|\cdot\|)$ satisfies the Prus-Szczepanik condition [(PSz) in short].

The following implications hold.

$$
\begin{gathered}
R(X)<2 \\
\varepsilon_{0}(X)<2 \Rightarrow M(X)>1 \Rightarrow(P S z) \Rightarrow \text { WFPP. }
\end{gathered}
$$

\subsection{Conditions depending on the dual space}

There are several results which establish the FPP for Banach spaces whose dual space satisfy suitable conditions. Maybe the following, due to Turret, is one of the earliest.

Theorem 3.16 [63, p. 283] If $X$ is a Banach space with $\varepsilon_{0}\left(X^{*}\right)<1$, then both $X$ and $X^{*}$ are superreflexive spaces and have normal structure.

Actually, the usefulness of this kind of result for a given Banach space, say $X$, depends on the knowledge of its dual space $X^{*}$, which is not always available.

For $\varepsilon \in(0,2)$, a subset $A$ of $X$ is said to be symmetrically $\varepsilon$-separated if the distance between any two distinct points of $A \cup(-A)$ is at least $\varepsilon$ and a Banach space $X$ is $O$-convex if the unit ball $B_{X}$ contains no symmetrically $(2-\varepsilon)$-separated subset of cardinality $n$ for some $\varepsilon>0$ and some $n \in \mathbb{N}$. $O$-convex Banach spaces are superreflexive. In 2008 Dowling et al. [12] proved the following fixed point result.

Theorem 3.17 If $X^{*}$ is $O$-convex, then the Banach space $X$ has the FPP.

Since $\varepsilon_{0}(X)<2$ if and only if $\varepsilon_{0}\left(X^{*}\right)<2$, uniformly nonsquare Banach spaces have $O$-convex dual, and then this theorem is a generalization of the Mazcuñán's result above reported.

Naidu and Sastry [48] also characterized the dual property to $O$-convexity. For $\varepsilon>0$, a convex subset $A$ of $B_{X}$ is an $\varepsilon$-flat if $A \cap(1-\varepsilon) B_{X}=\emptyset$. A collection $\mathcal{D}$ of $\varepsilon$-flats is jointly complemented (jcc in short) if, for each distinct $\varepsilon$-flats $A$ and $B$ in $\mathcal{D}$, the sets $A \cap B$ and $A \cap(-B)$ are nonempty. Define

$$
E(n, X)=\inf \left\{\varepsilon>0: B_{X} \text { contains a jcc of } \varepsilon-\text { flats of cardinality } n\right\} .
$$

A Banach space $X$ is said to be $E$-convex if $E(n, X)>0$ for some $n \in \mathbb{N}$. Since a Banach space is $E$-convex if and only if its dual space is $O$-convex, Theorem 3.17 can be restated as follows.

Theorem 3.18 E-convex Banach spaces have the FPP. 


\subsection{Weak orthogonality}

A Banach space $X$ has the (WORTH) property (Sims [55]) if, for all $x \in X$ and all weakly null sequence $\left(x_{n}\right)$ in $X$,

$$
\lim _{n}\left|\left\|x_{n}-x\right\|-\left\|x_{n}+x\right\|\right|=0 .
$$

If $X$ is a reflexive Banach space enjoying property (WORTH) and $R(X)<2$ then $X$ has NS (Sims [56]). If we define

$$
\mu(X):=\inf \left\{r>0: \lim \sup \left\|x_{n}+x\right\| \leq r \lim \sup \left\|x_{n}-x\right\|: x_{n} \rightarrow 0_{X} \quad x \in X\right\},
$$

then $X$ has property (WORTH) if and only if $\mu(X)=1$. The main result in [14] is the following.

Theorem 3.19 If $X$ is reflexive and $\mu(X)=1$, then $X$ enjoys FPP.

Although this result was an affirmative answer to a question raised by Sims [55], it is seemingly unknown if the above theorem remains true for $\mu(X)<b \in(1,3]$.

\subsection{Stability}

It is possible to prove FPP of a Banach space, by using that this space is isomorphic and close enough in the Banach-Mazur sense to another space which satisfies the FPP. This type of results is usually referred to as stability results.

For instance, for uniformly convex Banach spaces, Bynum [8] showed the following.

Theorem 3.20 If $(X,\|\cdot\|)$ is a unifomly convex Banach space and if $(Y,||$.$) is a Banach space such that$

$$
d(X, Y) \leq \mathrm{WCS}(X)
$$

then $Y$ has the FPP.

Here, the coefficient $W C S(X)$ is the supremum of the set of all numbers $M$ with the property that for each weakly convergent sequence $\left(x_{n}\right)$ with asymptotic diameter $A$, there is some $y$ in the closed convex hull of the (range of the) sequence such that $M \lim \sup _{n}\left\|x_{n}-y\right\| \leq A$.

The constant WCS $(X)$ has been very often considered to prove the WFPP of a Banach space. For example, $\operatorname{WCS}\left(\ell_{2},\|\cdot\|_{2}\right)=\sqrt{2}$ and

$$
\operatorname{WCS}\left(E_{\beta}\right)= \begin{cases}\frac{\sqrt{2}}{\beta} & 1 \leq \beta \leq \sqrt{2} \\ 1 & \sqrt{2}<\beta<\infty .\end{cases}
$$

According to the Bynum result, if the Banach-Mazur distance from $X$ to $\left(\ell_{2},\|\cdot\|_{2}\right)$ is less than $\sqrt{2}$, then $X$ has the FPP. However, there are several papers containing further improvements of this stability bound, which are due to Prus, Jiménez-Melado and Llorens-Fuster, García-Falset, Domínguez Benavides, Japón, Lin, and others (see the survey [22] for more details).

As far as the author knows, the deepest result for the stability of the FPP from Hilbert spaces is contained in the Eva Mazcuñán Navarro paper [51].

Theorem 3.21 If the Banach-Mazur distance from $X$ to $\left(\ell_{2},\|\cdot\|_{2}\right)$ is less than $\sqrt{\frac{5+\sqrt{17}}{2}}$, then $X$ has the fixed point property.

Some non-Hilbert renormings of $\ell_{2}$ can also transmit the FPP to spaces close enough. For instance, for the spaces $E_{\beta}$ one has that if $\|$.$\| is a norm on \ell_{2}$ such that for every $v \in \ell_{2}$

$$
|v|_{\beta} \leq\|v\| \leq b|v|_{\beta}
$$

with $b<\sqrt{2}$, then $\left(\ell_{2},\|\cdot\|\right)$ has the FPP (see [31]). 
Remark 3.22 Although Eva Mazcuñán's stability result gives a greater radius of propagation of the FPP than Bynum's Theorem 3.20 for Hilbert spaces, the latter theorem ensures stability from any uniformly convex renorming $X$ of $\ell_{2}$ because for such spaces $W C S(X)>1$ (in fact, it is well known than $\operatorname{WCS}(X) \geq \frac{1}{1-\delta_{X}(1)}$ for every Banach space. If $X$ is uniformly convex then $\left.\delta_{X}(1)>0\right)$.

On the other hand, if $\left(\ell_{2},\|\cdot\|_{b}\right)$ is a renorming of $\ell_{2}$, then it is a superreflexive Banach space. It is also well known that for every $\varepsilon>0$ there exists an equivalent uniformly convex norm $\|\cdot\|_{\varepsilon}$ on $\ell_{2}$ such that for every $x \in \ell_{2},\|x\|_{\varepsilon} \leq\|x\|_{b} \leq(1+\varepsilon)\|x\|_{\varepsilon}$. Therefore, by Bynum's theorem if

$$
d\left(\left(\ell_{2},\|\cdot\|_{\varepsilon}\right),\left(\ell_{2},\|\cdot\|_{b}\right)\right)<\operatorname{WCS}\left(\ell_{2},\|\cdot\|_{\varepsilon}\right)
$$

then $\left(\ell_{2},\|\cdot\|_{b}\right)$ has the FPP. Although the right-hand side of the above inequality has the lower bound $\frac{1}{1-\delta_{\left(\ell_{2},\|.\| \varepsilon\right)}(1)}>1$ and the left-hand side of the same inequality is very close to 1 , both sides are depending on $\varepsilon$ and we need more information in order to apply this Theorem for proving the FPP for $\left(\ell_{2},\|\cdot\|_{b}\right)$. It seems that this line of thinking has not been exploited.

Observe that, given the space $\left(\ell_{2},\|\cdot\|_{b}\right)$, the standard norm of $\ell_{2}$ is not necessarily the closest Hilbert norm to $\|\cdot\|_{b}$ and it is not usually clear what is this closest Hilbert norm.

\subsection{Conditions depending on a (Schauder) basis}

A Schauder basis $\left(e_{n}\right)$ of $(X,\|\cdot\|)$ is called unconditional if for every choice of signs $\theta=\left(\theta_{n}\right)$, one has that $\sum_{n=1}^{\infty} \theta_{n} a_{n} e_{n}$ is convergent provided $\sum_{n=1}^{\infty} a_{n} e_{n}$ is also convergent. In this case the number

$$
\mu:=\sup \left\{\left\|\sum_{n=1}^{\infty} \theta_{n} a_{n} e_{n}\right\|:\left\|\sum_{n=1}^{\infty} a_{n} e_{n}\right\|=1, \theta_{n}= \pm 1\right\}
$$

is called the unconditional constant of $\left(e_{n}\right)$.

If $\left(e_{n}\right)$ is an unconditional basis for $X$, then it is well known that for each nonempty $F \subset \mathbb{N}$, the projection

$$
P_{F}\left(\sum_{n=1}^{\infty} a_{n} e_{n}\right):=\sum_{n \in F} a_{n} e_{n}
$$

is well defined and the constant $c:=\sup \left\{\left\|P_{F}\right\|\right\}$ is finite. It is easy to verify that $1 \leq c \leq \mu \leq 2 c$. The basis $\left(e_{n}\right)$ is called strongly monotonous whenever $c=1$. Two sufficient conditions for WFPP in terms of these constants are well known:

Theorem 3.23 (Khamsi [37]) Let $(X,\|\cdot\|)$ be a reflexive Banach space with an unconditional basis such that

$$
c(\mu+2)<4 \text {. }
$$

Then, $(X,\|\cdot\|)$ has FPP.

Theorem 3.24 (Lin [45]) Let $(X,\|\cdot\|)$ be a super-reflexive Banach space with $c=1$. Then $(X,\|\cdot\|)$ has the FPP.

In order to apply Theorems 3.23 and 3.24, it must be noted that their assumptions are strongly dependent on the choice of a basis. For renormings of $\ell_{2}$ the standard basis $\left(e_{n}\right)$ is not the only possible choice.

Example 3.25 Let us observe that $E_{\beta}$ falls into the scope of the two theorems above since $c$ and $\mu$ are equal to 1 for the standard basis in this space.

On the other hand, one has, for the van Dulst renorming,

$$
\|(-1,1,1,0, \ldots)\|=1,\|(1,1,1,0, \ldots)\|=3 .
$$

Hence $\mu \geq 3$. Moreover for $F=\{2,3\} \subset \mathbb{N},\left\|P_{F}(-1,1,1,0, \ldots)\right\|=2$ which implies that $c \geq\left\|P_{F}\right\| \geq 2$. Therefore, with respect to the canonical basis, $c(2+\mu) \geq 10$. It is not clear whether these constants can be improved with respect to another basis.

Further fixed point results depending on some constants like $c$ and $\mu$, associated to a Schauder basis, can be found in [21]. 


\section{Independence of the four main sufficient conditions}

Very recently it has been realized that $E$-convex Banach spaces enjoy the Prus-Szczepanik condition (see [47]). Hence, after the results of the previous section, four purely geometrical properties, sufficient conditions for FPP in reflexive spaces, are in some sense maximal. These are ANS, OC, PSz, and WORTH. Next, we shall prove that these geometrical properties are pairwise independent. To do this, we will consider the following examples.

Example 4.1 Consider again, for $\beta \geq 1$, the spaces $E_{\beta}$ introduced in Example 3.1.

Some well-known properties of these spaces are the following.

(1) $\mu\left(E_{\beta}\right)=1$ for all $\beta \geq 1$ [55].

(2) The spaces $E_{\beta}$ have ANS if and only if $1 \leq \beta<2$, and they have NS if and only if $1 \leq \beta<\sqrt{2}$ (see [4]).

(3) The space $E_{\beta}$ is OC for every $\beta \geq 1$ (see [31]).

(4) $M\left(E_{\beta}\right)>1$ for every $\beta \geq 1$ (see [10]). Hence all the $E_{\beta}$ spaces satisfy (PS) condition.

Proposition 4.2 For $\beta \geq \sqrt{2}$, the space $E_{\beta}$ fails to be E-convex.

Proof From a result due to Saejung [54, Theorem 5], every $E$-convex Banach space $X$ with $\mu(X)=1$ has normal structure.

Since $\mu\left(E_{\beta}\right)=1$, and $E_{\beta}$ fails to have NS for $\beta \geq \sqrt{2}$, we would have a contradiction if $E_{\beta}$ were $E$-convex for some $\beta \geq \sqrt{2}$.

Notice that $\varepsilon_{0}\left(E_{\beta}\right)<2$ for $1 \leq \beta<\sqrt{2}$ see [27, p. 58] and hence the space $E_{\beta}$ is $E$-convex if and only if $\beta \in[1, \sqrt{2})$.

Example 4.3 Let $Y$ be the space $\ell_{2}$ endowed with the norm

$$
\|x\|:=\max \left\{\|x\|_{2}, \mathcal{M}(x)\right\},
$$

where for $x \in \ell_{2}$

$$
\mathcal{M}(x):=\sup \{|x(i)|+|x(j)|: 1 \leq i<j\} .
$$

It is straightforward to check that $Y$ has WORTH property, that is, that $\mu(Y)=1$.

Proposition 4.4 Y fails $O C$.

Proof For $n, m \in \mathbb{N}, n \neq m$,

$$
\left\|e_{n}\right\|=1, \quad\left\|e_{n}+e_{m}\right\|=\left\|e_{n}-e_{m}\right\|=2 .
$$

Then, $D\left[\left(e_{n}\right)\right]=2$. For $z=e_{n}+e_{m}$ one has $\|z\|=2$ and

$$
1=\left\|z-e_{n}\right\|=\left\|z-e_{m}\right\|=\frac{1}{2}\left\|e_{n}-e_{m}\right\| .
$$

Thus, $\forall \beta>0, z \in M_{\beta}\left(e_{m}, e_{n}\right)$ if $m \neq n$, and

$$
\left|M_{\beta}\left(e_{m}, e_{n}\right)\right| \geq\|z\|=2 .
$$

which implies

$$
\limsup _{n}\left(\limsup _{m}\left|M_{\beta}\left(e_{n}, e_{m}\right)\right|\right) \geq 2=D\left[\left(e_{n}\right)\right]
$$

Thus, $Y$ is not OC.

Proposition 4.5 The van Dulst space fails PSz condition 
Proof Take

$$
x_{n}=\left(0, \ldots, 0, \frac{4}{2}, \frac{1}{2}, 0, \ldots\right) .
$$

Note that $\left\|x_{n}\right\|=1$ and that, for $m>n$,

$$
\left\|x_{m}-x_{n}\right\|=\left\|\left(0, \ldots, 0,-\frac{1}{2},-\frac{1}{2}, 0, \ldots, 0, \frac{1}{2}, \frac{1}{2}, 0 \ldots\right)\right\|=1
$$

Then, $\left(x_{n}\right) \in \mathfrak{M}_{V D}:=\left\{\left(y_{n}\right): y_{n} \in B_{V D} n=1, \ldots, D\left(y_{n}\right) \leq 1, y_{n} \rightarrow 0_{\ell_{2}}\right\}$. Moreover, $e_{1} \in S_{V D}$, and for every $n \geq 1$,

$$
\left\|e_{1}+x_{n}\right\|=\left\|\left(1,0, \ldots, 0, \frac{1}{2}, \frac{1}{2}, \ldots\right)\right\|=2 .
$$

Therefore, for every $\varepsilon \in(0,1)$,

$$
\begin{aligned}
b_{1}\left(1, e_{1}\right): & =\sup _{\left(y_{n}\right) \in \mathfrak{M}_{V D}} \liminf _{n}\left\|e_{1}+y_{n}\right\|-\left\|e_{1}\right\| \\
& \geq \liminf _{n}\left\|e_{1}+x_{n}\right\|-\left\|e_{1}\right\| \\
& =2-1>1-\varepsilon
\end{aligned}
$$

In the same way, it is clear that $\left(-x_{n}\right) \in \mathfrak{N}_{V D}$. For $n>1$,

$$
\left\|e_{1}+\left(-x_{n}\right)\right\|=\left\|\left(1,0, \ldots,-\frac{1}{2},-\frac{1}{2}, 0, \ldots\right)\right\|=\max \left\{\frac{1}{3} \sqrt{\frac{3}{2}}, 1\right\}=1,
$$

and

$$
d\left(1, e_{1}\right):=\inf _{\left(y_{n}\right) \in \mathfrak{N}_{V D}} \limsup _{n}\left\|e_{1}+y_{n}\right\|-\left\|e_{1}\right\| \leq \limsup _{n}\left\|e_{1}-x_{n}\right\|-\|v\|=0<\varepsilon
$$

Proposition 4.6 $\mu(V D) \geq 2$.

Proof Take $x_{n}:=\left(0, \ldots, 0, \stackrel{(2 n+1)}{\frac{1}{2}}, \frac{1}{2}, 0, \ldots\right)$, one has $x_{n} \rightarrow 0_{\ell_{2}}$ and $\left\|x_{n}\right\|=1(n=1,2, \ldots)$. Since $e_{1} \in S_{V D}$, and for $n>1$,

$$
\begin{aligned}
\left\|e_{1}+x_{n}\right\| & =\left\|\left(1,0, \ldots, 0, \frac{1}{2}, \frac{1}{2}, 0, \ldots\right)\right\|=2 . \\
\left\|e_{1}-x_{n}\right\| & =\left\|\left(1,0, \ldots, 0, \frac{-1}{2}, \frac{-1}{2}, 0, \ldots\right)\right\|=1 .
\end{aligned}
$$

It follows that $\mu(V D) \geq 2$.

Example 4.7 The Banach space $\ell_{2} \oplus_{1} \ell_{2}$.

This space is UNC and hence it satisfies the PS condition (see [52]). Notice that this space is isometric to $\left(\ell_{2},\|\cdot\|\right)$ for a suitable renorming $\|\cdot\|$.

Proposition 4.8 The space $\ell_{2} \oplus_{1} \ell_{2}$ is E-convex but it fails to be $O C$.

Proof (See [16]) Indeed, $\left(\ell_{2},\|\cdot\|_{2}\right)$ is $P$-convex, and from Theorem 1.5 in [6] $P$-convexity is preserved under $\ell_{\infty}$-direct sums. Then, $\ell_{2} \oplus_{1} \ell_{2}=\left(\ell_{2} \oplus_{1} \ell_{2}\right)^{*}$ is $P$-convex and hence $O$-convex. To see that this space fails to be $\mathrm{OC}$, for $k$ positive integer put

$$
v_{2 k}=\left(0_{\ell_{2}}, e_{2 k}\right), \quad v_{2 k+1}=\left(e_{2 k+1}, 0_{\ell_{2}}\right) .
$$

It is obvious that the sequence $\left(v_{n}\right)$ is weakly convergent to $\left(0_{\ell_{2}}, 0_{\ell_{2}}\right)$, and that $\left\|v_{n}\right\|=1$ for $n=1,2, \ldots$. 
For $n<m$ one has that $\left\|v_{n}-v_{m}\right\|=2$ whenever $n$ and $m$ have different parity while $\left\|v_{n}-v_{m}\right\|=1$ if $m$ and $n$ have the same parity. Thus, $D\left(v_{n}\right)=\lim \sup _{m}\left[\lim \sup _{n}\left\|v_{m}-v_{n}\right\|\right]=2$. If $\beta>0$ and $m, n$ have different parity, since

$$
\left\|\left(v_{m}+v_{n}\right)-v_{n}\right\|=1=\frac{1}{2}\left\|v_{m}-v_{n}\right\|, \quad\left\|\left(v_{m}+v_{n}\right)-v_{m}\right\|=1=\frac{1}{2}\left\|v_{m}-v_{n}\right\|,
$$

we have $w_{m, n}=v_{m}+v_{n} \in M_{\beta}\left(v_{n}, v_{m}\right)$. Given that $\left\|w_{m, n}\right\|=\left\|v_{n}+v_{m}\right\|=\left\|e_{n}\right\|_{2}+\left\|e_{m}\right\|_{2}=2$, then

$$
\left|M_{\beta}\left(v_{n}, v_{m}\right)\right| \geq\left\|w_{m, n}\right\|=2
$$

and therefore for every $\beta>0$,

$$
\limsup _{m}\left[\limsup _{n}\left|M_{\beta}\left(v_{n}, v_{m}\right)\right|\right] \geq 2=D\left(v_{n}\right),
$$

which implies that $\ell_{2} \oplus_{1} \ell_{2}$ fails to be OC.

Example 4.9 The Bynum spaces.

For $x \in \ell_{2}$ let

$$
\|x\|_{2,1}:=\left\|x^{+}\right\|_{2}+\left\|x^{-}\right\|_{2}, \quad\|x\|_{2, \infty}:=\max \left\{\left\|x^{+}\right\|_{2},\left\|x^{-}\right\|_{2}\right\} .
$$

The spaces $\ell_{2,1}:=\left(\ell_{2},\|\cdot\|_{2,1}\right)$ and $\ell_{2, \infty}:=\left(\ell_{2},\|\cdot\|_{2, \infty}\right)$ were introduced by Bynum.

Well-known features of these spaces are the following:

(1) $\left(\ell_{2},\|\cdot\|_{2,1}\right)$ has NS [and hence ANS] [27].

(2) $\left(\ell_{2},\|\cdot\|_{2,1}\right)^{*}=\left(\ell_{2},\|\cdot\|_{2, \infty}\right)$.

(3) The space $\ell_{2, \infty}$ fails to have ANS (Bynum, private communication).

(4) $\varepsilon_{0}\left(\ell_{2, \infty}\right)=1$. Hence $M\left(\ell_{2, \infty}\right)>1$ and this space satisfy PS condition. $\varepsilon_{0}\left(\ell_{2, \infty}\right)=1$ also it directly implies that $\ell_{2, \infty}$ is $E$-convex.

(5) $\ell_{2, \infty}$ is OC (see [29]).

(6) $\mu\left(\ell_{2,1}\right)=\mu\left(\ell_{2, \infty}\right)=\sqrt{2}$ (see [35]).

Example 4.10 Let $V$ be the space $\ell_{2}$ endowed with the norm

$$
\|x\|_{V}:=\max \left\{\|x\|_{2}, \sqrt{2} A(x)\right\}
$$

where $A(x):=\sup \left\{\left|x_{1}+x_{n}\right|: n \geq 2\right\}$.

Proposition 4.11 The space $V$ fails PS condition.

Proof For each positive integer $n$ put $v_{n}:=\frac{1}{\sqrt{2}} e_{n}$. Note that $\left\|v_{n}\right\|_{V}=1$ and that, for $m>n>1$,

$$
\left\|v_{m}-v_{n}\right\|_{V}=1 \text {. }
$$

Then $\left(v_{n}\right) \in \mathfrak{M}_{V}:=\left\{\left(y_{n}\right): y_{n} \in B_{V} n=1, \ldots, D\left(y_{n}\right) \leq 1, y_{n} \rightarrow 0_{\ell_{2}}\right\}$. Moreover, $v_{1} \in S_{V}$, and for every $n \geq 1$,

$$
\left\|v_{1}+v_{n}\right\|_{V}=2 .
$$

Therefore, for every $\varepsilon \in(0,1)$,

$$
\begin{aligned}
b_{1}\left(1, v_{1}\right) & :=\sup _{\left(y_{n}\right) \in \mathfrak{M}_{V}} \liminf _{n}\left\|v_{1}+y_{n}\right\|_{V}-\left\|v_{1}\right\|_{V} \\
& \geq \liminf _{n}\left\|v_{1}+v_{n}\right\|_{V}-\left\|v_{1}\right\|_{V}=2-1>1-\varepsilon .
\end{aligned}
$$

In the same way, since $\left(-v_{n}\right) \in \mathfrak{N}_{V}$, and for $n \geq 2$,

$$
\left\|v_{1}+\left(-v_{n}\right)\right\|_{V}=\left\|\left(\frac{1}{\sqrt{2}}, 0, \ldots, 0,-\frac{1}{\sqrt{2}}, 0, \ldots\right)\right\|_{V}=\max \left\{1, \sqrt{2} \frac{1}{\sqrt{2}}\right\}=1
$$

and

$$
\begin{aligned}
d\left(1, v_{1}\right) & :=\inf _{\left(y_{n}\right) \in \mathfrak{N}_{V}} \lim \sup _{n}\left\|v_{1}+y_{n}\right\|_{V}-\left\|v_{1}\right\|_{V} \\
& \leq \lim \sup _{n}\left\|v_{1}+\left(-v_{n}\right)\right\|_{V}-\left\|v_{1}\right\|_{V}=0<\varepsilon .
\end{aligned}
$$


To prove that the space $V$ has asymptotic normal structure, we closely follow Lemma 3 in [4]. First, we need the following result, also from [4].

Lemma 4.12 Let $K$ a closed bounded and convex subset of $\ell_{2}$ and let $\left(x_{n}\right)$ be a sequence in $K$. Then, there exists a unique point $z \in K$ (called the $\|\cdot\|_{2}$-asymptotic-center of $\left(x_{n}\right)$ in $K$ ) which minimizes the functional $x \mapsto \limsup _{n \rightarrow \infty}\left\|x_{n}-x\right\|_{2}$. This point satisfies

(a)

$$
\limsup _{n \rightarrow \infty}\left\|x_{n}-z\right\|_{2}^{2}+\|z-x\|_{2}^{2} \leq \limsup _{n \rightarrow \infty}\left\|x_{n}-x\right\|_{2}^{2}
$$

for all $x \in K$, and

(b)

$$
2 \limsup _{n \rightarrow \infty}\left\|x_{n}-z\right\|_{2}^{2} \leq \limsup _{p \rightarrow \infty}\left(\limsup _{n \rightarrow \infty}\left\|x_{n}-x_{p}\right\|_{2}^{2}\right) .
$$

Proposition 4.13 $V$ has asymptotic normal structure.

Proof Assume for a contradiction that there exists a closed, bounded and convex subset $K$ of $\ell_{2}$, with $\operatorname{diam}_{\|\cdot\|_{V}}(K)=d>0$ and a sequence $\left(x_{n}\right)$ in $K$, with $x_{n}-x_{n+1} \rightarrow 0_{\ell_{2}}$, and such that for every $x \in K$

$$
\left\|x_{n}-x\right\|_{V} \rightarrow d .
$$

Since $K$ is weakly compact we can suppose, passing to a subsequence if necessary, that $x_{n} \rightarrow x \in K$. Let $z \in K$ be the $\|\cdot\|_{2}$-asymptotic-center of $\left(x_{n}\right)$ in $K$. We claim that $z=x$.

Indeed, for every positive integer $n$ we have

$$
\left\|x_{n}-z\right\|_{2}^{2}=\left\|x_{n}-x\right\|_{2}^{2}+\|x-z\|_{2}^{2}-2\left\langle x_{n}-x, x-z\right\rangle .
$$

Since $x_{n}-x \rightarrow 0$

$$
\limsup _{n \rightarrow \infty}\left\|x_{n}-z\right\|_{2}^{2}=\limsup _{n \rightarrow \infty}\left\|x_{n}-x\right\|_{2}^{2}+\|x-z\|_{2}^{2} .
$$

On the other hand, from Lemma 4.12(a) we have

$$
\limsup _{n \rightarrow \infty}\left\|x_{n}-z\right\|_{2}^{2}+\|x-z\|_{2}^{2} \leq \limsup _{n \rightarrow \infty}\left\|x_{n}-x\right\|_{2}^{2} .
$$

Bearing in mind (1) from this last inequality we get that

$$
\limsup _{n \rightarrow \infty}\left\|x_{n}-z\right\|_{2}^{2}+\|x-z\|_{2}^{2} \leq \limsup _{n \rightarrow \infty}\left\|x_{n}-x\right\|_{2}^{2}-\|x-z\|_{2}^{2}
$$

and hence $\|x-z\|_{2}=0$, that is, $z=x$ as we claimed.

By Lemma 4.12(b) we have $\lim \sup _{n \rightarrow \infty}\left\|x_{n}-z\right\|_{2}^{2} \leq \frac{d^{2}}{2}$ and since $\left\|x_{n}-z\right\|_{V}^{2} \rightarrow d^{2}$ we have $\left[\sqrt{2} A\left(x_{n}-\right.\right.$ $z)]^{2}$

$\rightarrow d^{2}$, that is,

$$
\sup _{j \geq 2}\left|x(1)+x_{n}(j)-z(1)-z(j)\right| \rightarrow \frac{d}{\sqrt{2}}
$$

Given that $x_{n}, z \in \ell_{2}$, we have that the above supremum is attained, that is, for each positive integer $n$ there exists a positive integer $j_{n}$ such that

$$
A\left(x_{n}-z\right)=\left|x(1)+x_{n}\left(j_{n}\right)-z(1)-z\left(j_{n}\right)\right| .
$$

Since $x_{n}-z \rightarrow 0_{\ell_{2}}$, and hence $x_{n}(1)-z(1) \rightarrow 0$, and $x_{n}-x_{n+1} \rightarrow 0_{\ell_{2}}$,

$$
\begin{aligned}
& A\left(x_{n}-z\right)^{2}-2\left|x_{n}(1)-z(1)\right| \cdot\left|x_{n}\left(j_{n}\right)-z\left(j_{n}\right)\right| \\
& \quad+\left(A\left(x_{n+1}-z\right)-A\left(x_{n}-x_{n+1}\right)-\left|x_{n}(1)-z(1)\right|\right)^{2} \longrightarrow d^{2} .
\end{aligned}
$$


Therefore, given $r \in\left(\frac{d^{2}}{2}, d^{2}\right)$ there exists a positive integer $n_{0}$ such that for every $n \geq n_{0}$ we have

$$
\begin{aligned}
& \left\|x_{n}-z\right\|_{2}^{2} \leq r \\
& A\left(x_{n+1}-z\right)-A\left(x_{n}-x_{n+1}\right)-\left|x_{n}(1)-z(1)\right| \geq 0
\end{aligned}
$$

and

$$
\begin{aligned}
& A\left(x_{n}-z\right)^{2}-2\left|x_{n}(1)-z(1)\right|\left|x_{n}\left(j_{n}\right)-z\left(j_{n}\right)\right| \\
& \quad+\left(A\left(x_{n+1}-z\right)-A\left(x_{n}-x_{n+1}\right)-\left|x_{n}(1)-z(1)\right|\right)^{2}>r .
\end{aligned}
$$

Put $k=j_{n_{0}}$. We claim that $j_{n}=k$ for every $n \geq n_{0}$. Otherwise there exists $n \geq n_{0}$ such that $j_{n} \neq j_{n+1}$ and then

$$
\begin{aligned}
r & \geq \mid x_{n}-z \|_{2}^{2} \\
& \geq\left|x_{n}(1)-z(1)\right|^{2}+\left|x_{n}\left(j_{n}\right)-z\left(j_{n}\right)\right|^{2}+\left|x_{n}\left(j_{n+1}\right)-z\left(j_{n+1}\right)\right|^{2} \\
& \geq\left|x_{n}(1)-z(1)+x_{n}\left(j_{n}\right)-z\left(j_{n}\right)\right|^{2}-2\left|x_{n}(1)-z(1)\right|\left|x_{n}\left(j_{n}\right)-z\left(j_{n}\right)\right| \\
& +\left|x_{n}\left(j_{n+1}\right)-z\left(j_{n+1}\right)\right|^{2} \\
& =\left|x_{n}(1)-z(1)+x_{n}\left(j_{n}\right)-z\left(j_{n}\right)\right|^{2}-2\left|x_{n}(1)-z(1)\right|\left|x_{n}\left(j_{n}\right)-z\left(j_{n}\right)\right| \\
& +\mid x_{n+1}\left(j_{n+1}\right)-z\left(j_{n+1}\right)+x_{n+1}(1)-z(1)+ \\
& +x_{n}\left(j_{n+1}\right)-x_{n+1}\left(j_{n+1}\right)+x_{n}(1)-x_{n+1}(1)-x_{n}(1)+\left.z(1)\right|^{2}
\end{aligned}
$$

Since $A\left(x_{n+1}-z\right)-A\left(x_{n}-x_{n+1}\right)-\left|x_{n}(1)-z(1)\right| \geq 0$, that is

$$
\left|x(1)+x_{n+1}\left(j_{n+1}\right)-z(1)-z\left(j_{n+1}\right)\right|-\left|x(1)+x_{n}\left(j_{n}\right)-z(1)-z\left(j_{n}\right)\right|-\left|x_{n}(1)-z(1)\right| \geq 0
$$

then,

$$
\begin{aligned}
0 \leq & \left|x(1)+x_{n+1}\left(j_{n+1}\right)-z(1)-z\left(j_{n+1}\right)\right| \\
& -\left|x(1)+x_{n}\left(j_{n}\right)-z(1)-z\left(j_{n}\right)\right|-\left|x_{n}(1)-z(1)\right| \\
\leq & \left|x(1)+x_{n+1}\left(j_{n+1}\right)-z(1)-z\left(j_{n+1}\right)\right| \\
& -\left|\left(x(1)+x_{n}\left(j_{n}\right)-z(1)-z\left(j_{n}\right)\right)+\left(x_{n}(1)-z(1)\right)\right| \\
\leq & \mid x(1)+x_{n+1}\left(j_{n+1}\right)-z(1)-z\left(j_{n+1}\right) \\
& -\left(x(1)+x_{n}\left(j_{n}\right)-z(1)-z\left(j_{n}\right)\right)-\left(x_{n}(1)-z(1)\right) \mid
\end{aligned}
$$

and hence

$$
\begin{aligned}
0 \leq & \left(\left|x(1)+x_{n+1}\left(j_{n+1}\right)-z(1)-z\left(j_{n+1}\right)\right|\right. \\
& \left.-\left|x(1)+x_{n}\left(j_{n}\right)-z(1)-z\left(j_{n}\right)\right|-\left|x_{n}(1)-z(1)\right|\right)^{2} \\
\leq & \mid x(1)+x_{n+1}\left(j_{n+1}\right)-z(1)-z\left(j_{n+1}\right) \\
& -\left(x(1)+x_{n}\left(j_{n}\right)-z(1)-z\left(j_{n}\right)\right)-\left.\left(x_{n}(1)-z(1)\right)\right|^{2} .
\end{aligned}
$$

Bearing this in mind, it follows from (2) that

$$
\begin{aligned}
r \geq & \left|x_{n}(1)-z(1)+x_{n}\left(j_{n}\right)-z\left(j_{n}\right)\right|^{2}-2\left|x_{n}(1)-z(1)\right|\left|x_{n}\left(j_{n}\right)-z\left(j_{n}\right)\right| \\
& +\mid x_{n+1}\left(j_{n+1}\right)-z\left(j_{n+1}\right)+x_{n+1}(1)-z(1)+ \\
& +x_{n}\left(j_{n+1}\right)-x_{n+1}\left(j_{n+1}\right)+x_{n}(1)-x_{n+1}(1)-x_{n}(1)+\left.z(1)\right|^{2} \\
\geq & \left|x_{n}(1)-z(1)+x_{n}\left(j_{n}\right)-z\left(j_{n}\right)\right|^{2}-2\left|x_{n}(1)-z(1)\right|\left|x_{n}\left(j_{n}\right)-z\left(j_{n}\right)\right| \\
& +\left(\left|x(1)+x_{n+1}\left(j_{n+1}\right)-z(1)-z\left(j_{n+1}\right)\right|\right. \\
& \left.-\left|x(1)+x_{n}\left(j_{n}\right)-z(1)-z\left(j_{n}\right)\right|-\left|x_{n}(1)-z(1)\right|\right)^{2} \\
= & \left|x_{n}(1)-z(1)+x_{n}\left(j_{n}\right)-z\left(j_{n}\right)\right|^{2}-2\left|x_{n}(1)-z(1)\right|\left|x_{n}\left(j_{n}\right)-z\left(j_{n}\right)\right| \\
& +\left(A\left(x_{n+1}-z\right)-A\left(x_{n}-x_{n+1}\right)-\left|x_{n}(1)-z(1)\right|\right)^{2}>r,
\end{aligned}
$$


a contradiction which proves our claim. Then $j_{n}=k$ for every $n \geq n_{0}$. Since $x_{n}-z \rightarrow 0$ we have $x_{n}(1)-$ $z(1) \rightarrow 0$ and $x_{n}(k)-z(k) \rightarrow 0$ as $n \rightarrow \infty$. From this we obtain

$$
\begin{aligned}
\frac{d^{2}}{2} & =\lim _{n \rightarrow \infty} A\left(x_{n}-z\right)^{2} \\
& =\lim _{n \rightarrow \infty}\left|x_{n}(1)+x_{n}\left(j_{n}\right)-z(1)-z\left(j_{n}\right)\right|^{2} \\
& =\lim _{n \rightarrow \infty}\left|x_{n}(1)+x_{n}(k)-z(1)-z(k)\right|^{2}=0 .
\end{aligned}
$$

Consequently, $d=0$, which contradicts our assumption.

Example 4.14 Let $\beta \geq 1$. Let us consider the Banach space $X_{2, \beta}:=\left(\mathbb{R} \oplus_{1} E_{\beta}\right) \oplus_{\infty} \ell_{2}$.

Proposition 4.15 For $\beta \geq \sqrt{2}$ the space $X_{2, \beta}$ enjoys property WORTH but it fails PS condition.

Proof Consider the sequence $\left(w_{n}\right)$ in $E_{\beta}$ given by $w_{n}=\frac{1}{\beta} e_{n}$. Then, take the sequences $\left(z_{m}\right)$ and $\left(y_{m}\right)$ in $S_{X_{2, \beta}}$ defined as

$$
z_{m}=\left(\left(0, w_{m}\right), 0_{\ell_{2}}\right), \quad y_{m}=\left(\left(0,0_{E_{\beta}}\right), e_{m}\right)
$$

For $x=\left(\left(1,0_{E_{\beta}}\right), 0_{\ell_{2}}\right) \in S_{X_{2, \beta}}$ and positive integers $m, n$ we have that

$$
\begin{aligned}
& \left\|x+z_{m}\right\|_{X_{2, \beta}}=\left\|\left(\left(1, w_{m}\right), 0_{\ell_{2}}\right)\right\|_{X_{2, \beta}}=\max \left\{1+\left|w_{m}\right|_{\beta}, 0\right\}=2 \\
& \left\|x+y_{m}\right\|_{X_{2, \beta}}=\left\|\left(\left(1,0_{E_{\beta}}\right), e_{m}\right)\right\|_{X_{2, \beta}}=\max \left\{1,\left\|e_{m}\right\|_{p}\right\}=1 \\
& \left\|z_{n}-z_{m}\right\|_{X_{2, \beta}}=\left\|\left(\left(0, w_{m}-w_{n}\right), 0_{\ell_{2}}\right)\right\|_{X_{2, \beta}}=\left|w_{n}-w_{m}\right|_{\beta}=1
\end{aligned}
$$

Since $z_{m} \rightarrow 0_{X_{2, \beta}}$ and $y_{m} \rightarrow 0_{X_{2, \beta}}$ we obtain that $\left(z_{n}\right) \in \mathfrak{M}_{X_{2, \beta}}$ and $\left(y_{n}\right) \in \mathfrak{N}_{X_{2, \beta}}$. Therefore, we have for every $\varepsilon \in(0,1)$ that

$$
d(1, x)=\inf _{\left(\xi_{n}\right) \in \mathfrak{N}_{X}} \limsup _{n \rightarrow \infty}\left\|x+\xi_{n}\right\|_{X_{2, \beta}}-1 \leq \limsup _{n \rightarrow \infty}\left\|x+y_{n}\right\|_{X_{2, \beta}}-1=0 \leq \varepsilon
$$

and

\begin{tabular}{|c|c|c|c|c|}
\hline 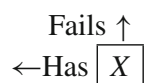 & ANS & $\mathrm{OC}$ & PSz & WORTH \\
\hline ANS & $*$ & $Z$ & $V$ & $\ell_{2,1}$ \\
\hline $\mathrm{OC}$ & $V D$ & $*$ & $V D$ & $\ell_{2, \infty}$ \\
\hline & $\ell_{2, \infty}$ & $\ell_{2} \oplus_{1} \ell_{2}$ & & $\ell_{2, \infty}$ \\
\hline WORTH & $E_{\beta}, \beta \geq 2$ & $Y$ & $X_{2, \beta}$ & $*$ \\
\hline
\end{tabular}

$$
b_{1}(1, x)=\sup _{\left(\xi_{n}\right) \in \mathfrak{M}_{X}} \liminf _{n \rightarrow \infty}\left\|x+\xi_{n}\right\|_{X_{2, \beta}}-1 \geq \liminf _{n \rightarrow \infty}\left\|x+z_{n}\right\|_{X_{3, \beta}}-1=1 \geq 1-\varepsilon
$$

Thus, $X_{2, \beta}$ fails condition PS.

On the other hand, $X_{2, \beta}$ has property WORTH because it is well known that the spaces $\ell_{2}$ and $E_{\beta}$ have property WORTH and Kato and Tamura proved in [32] that this property is preserved under $\ell_{q}^{n}$-direct sums for $1 \leq q \leq \infty$.

\subsection{Summary}

Next, we summarize the above results in the following table: 


\section{Some pathological examples}

In $[27$, p. 65$]$ is said that,

...it is very unusual for spaces to fail to have normal structure...

Then, it seems even more unusual to fail to have each one of the above referred properties ANS, OC, PSz and WORTH. But very recently in [16] it has been shown that the space $W:=V D \oplus_{1} V D$ enjoys the FPP but it lacks these four geometrical sufficient conditions for FPP.

In the same way, in [34] a family of renormings of $\ell_{2}$ was introduced as follows.

For $x=(x(n)) \in \ell_{2}$ define

$$
\mathcal{M}(x):=\sup \{|x(2 i-1)|+|x(2 j)|: i, j \in \mathbb{N}\},
$$

and

$$
\mathcal{S}(x):=\sup \{|x(1)+x(n)+x(n+1)+x(n+2)|: n \geq 2\} .
$$

For $a>0$ and $b, c, d \geq 0$, let $X_{(a, b, c, d)}$ be the Banach space $\left(\ell_{2},\|\cdot\|\right)$, where the norm $\|\cdot\|$ is defined by

$$
\|x\|:=\max \left\{a\|x\|_{2}, b \mathcal{M}(x), c \mathcal{S}(x), d\|x\|_{\infty}\right\} .
$$

The norm $\|\cdot\|$ is equivalent to $\|\cdot\|_{2}$, since for every $x \in \ell_{2}$ we have

$$
a\|x\|_{2} \leq\|x\| \leq m\|x\|_{2},
$$

where $m=\max \{a, b \sqrt{2}, 2 c, d\}$.

The main result in [34] established just that $X_{(a, b, c, d)}$ enjoys the FPP. In particular, this is true for the space $\tilde{X}=X_{\left(\frac{1}{3}, 1,1,0\right)}$. In [34] it was also shown that $\tilde{X}$ separately fails ANS, OC, PSz and WORTH, that is, all the sufficient conditions for the FPP considered in the above section. Moreover, the Banach-Mazur distance between $\tilde{X}$ and $\left(\ell_{2},\|\cdot\|_{2}\right)$ is greater than Mazcuñán's stability bound, and finally, with respect to the standard basis $\left(e_{n}\right), c(\mu+2) \geq 6$, so Khamsi Theorem 3.23 does not work in this case.

\section{Final remarks}

The above examples in Sect. 5 are not quite sophisticated. One might think that the list of sufficient conditions for the FPP is not large enough. Then, those readers guessing that the answer to problem $[\mathrm{P}]$ is affirmative should search for either wider geometrical sufficient conditions or for very different techniques of those used until now. In any case, in $[2,48]$ several classes of Banach spaces lying between the class of uniformly nonsquare Banach spaces and the class of superreflexive spaces (also known as $J$-convex spaces) were studied. In particular, it seem to be unknown whether either $P$-convex, $Q$-convex or $O$-convex Banach spaces have the FPP.

On the other hand, if Problem [P] has a negative answer, that is, if there exists a renorming of $\ell_{2}$ lacking of the FPP, say $\left(\ell_{2},\|\cdot\|_{b}\right)$, then there exists a weakly compact convex subset $C$ od $\ell_{2}$ and a $\|\cdot\|_{b}$-nonexpansive fixed point free mapping $T: C \rightarrow C$. Without loss of generality we might assume in this situation that there exists a positive constant, say $\beta$, such that for every $v \in \ell_{2}$

$$
\|v\|_{2} \leq\|v\|_{b} \leq \beta\|v\|_{2} .
$$

After a dilatation and a translation if necessary, we may suppose also that $C \subset B_{2}$. Since $T$ would be $\|\cdot\|_{b^{-}}$ nonexpansive for every $x, y \in C$ and every positive integer $n$,

$$
\left\|T^{n}(x)-T^{n}(y)\right\|_{2} \leq\left\|T^{n}(x)-T^{n}(y)\right\|_{b} \leq\|x-y\|_{b} \leq \beta\|x-y\|_{2} .
$$

In other words, the mapping $T$ would leave invariant a subset $C$ of the Euclidean unit ball, it would be fixed point free, and moreover it would be $\|\cdot\|_{2}-\beta$-uniformly Lipschitzian on $C$, that is all iterates of $T$ would have $\beta$ as a common Lipschitz constant with respect to the norm $\|\cdot\|_{2}$. Thus there would exists a $\|\cdot\|_{2}-\beta$-uniformly Lipschitzian fixed point free and leaving invariant a closed convex subset $C$ of $B_{2}$. In particular, for $C=B_{2}$ several mappings fulfilling all these requirements are known. But, unfortunately, no renorming of $\ell_{2}$ exist such that these mappings become nonexpansive. See [46] for detailed information about this approach. 
Acknowledgments Research partially supported by a Grant from Ministerio de Educación y Cultura (Spain) MTM2009-10696C02-02.

Open Access This article is distributed under the terms of the Creative Commons Attribution License which permits any use, distribution, and reproduction in any medium, provided the original author(s) and the source are credited.

\section{References}

1. Alspach, D.E.: A fixed point free nonexpansive map. Proc. Am. Math. Soc. 82(3), 423-424 (1981)

2. Amir, D.; Franchetti, C.: The radius ratio and convexity properties in normed linear spaces. Trans. Am. Math. Soc. 282, 275-291 (1984)

3. Ayerbe Toledano, J.; Domínguez Benavides, T.; López Acedo, G.: Measures of Noncompactness in Metric Fixed Point Theory, vol. 99. Birkhäuser, Op. Th., Basel (1997)

4. Baillon, J.B.; Schöneberg, R.: Asymptotic normal structure and fixed points of nonexpansive mappings. Proc. Am. Math. Soc. 81, 257-264 (1981)

5. Belluce, L.P.; Kirk, W.A.; Steiner, E.F.: Normal structure in Banach spaces. Pac. J. Math. 26, $433-440$ (1968)

6. Brown, D.R.: $P$-convexity and $B$-convexity in Banach spaces. Trans. Am. Math. Soc. 187, 77-81 (1974)

7. Büber, T.; Kirk, W.A.: Constructive aspects of Fixed Point Theory for nonexpansive mappings. World Congress of Nonlinear Analysts '92, vols. I-IV (Tampa, FL, 1992), pp. 2115-2125. de Gruyer, Berlin

8. Bynum, W.L.: Normal structure coefficients for normal structure for Banach spaces. Pac. J. Math. 86, 427-436 (1980)

9. Domínguez Benavides, T.: Stability of the fixed point property for nonexpansive mappings. Houston J. Math. 22, 835-849 (1996)

10. Domínguez Benavides, T.; Japón Pineda, M.A.: Stability of the fixed point property for nonexpansive mappings in some classes of spaces. Comm. Appl. Nonlinear Anal. 5, 37-46 (1998)

11. Dowling, P.N.; Lennard, C.J.: Every nonreflexive subspace of $L_{1}[0,1]$ fails the fixed point property. Proc. Am. Math. Soc. 125, 443-446 (1997)

12. Dowling, P.N.; Randrianantoanina, B; Turett, B.: The fixed point property via dual space properties. J. Funct. Anal. 255, 768-775 (2008)

13. Fetter, H.; Gamboa de Buen, B.: $(r, k, l)$-somewhat uniformly noncreasy Banach spaces. International Conference on Fixed Point Theory and Applications, pp. 71-80. Yokohama Publications, Yokohama (2004)

14. Fetter, H.; Gamboa de Buen, B.: Properties WORTH and WORTH*, $(1+\delta)$ embeddings in Banach spaces with 1-unconditional basis and wFPP. Fixed Point Theory Appl. Art. ID 342691 (2010)

15. Fetter, H.; Gamboa de Buen, B.; García Falset, J.: Banach spaces which are somewhat uniformly noncreasy. J. Math. Anal. Appl. 285, 444-455 (2003)

16. Fetter Nathansky, H.; Llorens-Fuster, E.: A product space with the fixed point property. Fixed Point Theory Appl. 2012, 91 (2012)

17. García-Falset, J.: Banach spaces satisfying the fixed point property for nonexpansive mappings. Ph. D. Thesis, Univ. of Valencia, Spain (1990)

18. García-Falset, J.: Fixed point property in Banach spaces whose characteristic of convexity is less than 2. J. Austral. Math. Soc. Ser A. 54, 169-173 (1993)

19. García-Falset, J.: Stability and fixed points for nonexpansive mappings. Houston J. Math. 20, $495-505$ (1994)

20. García-Falset, J.: The fixed point property in Banach spaces with NUS property, J. Math. Anal. Appl. 215, 532-542 (1997)

21. García-Falset, J.; Llorens Fuster, E.: A geometric property of banach spaces related to the fixed point property. J. Math. Anal. Appl. 172, 39-52 (1993)

22. García-Falset, J.; Jiménez-Melado, A.; Llorens-Fuster, E.: Stability of the fixed point property for nonexpansive mappings. In: Kirk, W.A.; Sims, B. (eds.) Handbook of Metric Fixed Point Theory, pp. 231-238. Kluwer Academic Publications (2001)

23. García-Falset; J., Llorens Fuster, E.; Mazcunán Navarro, E. Banach spaces which are $r$-uniformly noncreasy. Nonlinear Anal. 53, 957-975 (2003)

24. García-Falset; J., Llorens Fuster, E.; Mazcuñán Navarro, E.: Uniformly nonsquare Banach spaces have the fixed point property for nonexpansive mappings. J. Funct. Anal. 233, 494-514 (2006)

25. Giles, J.R.; Sims, B.; Swaminathan, S.: A geometrically aberrant Banach space with normal structure. Bull. Austal. Math. Soc. 31, 75-81 (1985)

26. Goebel, K.: Convexivity of balls and fixed-point theorems for mappings with nonexpansive square. Compositio Math. 22, 269-274 (1970)

27. Goebel, K.; Kirk W.A.: Topics in Metric Fixed Point Theory. Cambridge University Press (1990)

28. Gossez, J.-P.; Lami Dozo, E.: Some geometric properties related to the fixed point theory for nonexpansive mappings. Pac. J. Math. 40, 565-573 (1972)

29. Jiménez-Melado, A.: Una propiedad geométrica de los espacios de Banach relacionada con la Teoría del Punto Fijo. Ph.D. dissertation, Univ. de Málaga (Spain) (1988)

30. Jiménez-Melado, A.: Stability of weak normal structure in James quasi reflexive space. Bull. Austral. Math. Soc. 46(3), 367-372 (1992)

31. Jiménez-Melado, A.; Llorens-Fuster, E.: A sufficient condition for the fixed point property. Nonlinear Anal. 20, 849-853 (1993)

32. Kato, M.; Tamura, T.: Weak nearly uniform smoothness and worth property of $\psi$-direct sums of Banach spaces $X \oplus_{\psi} Y$, Comment. Math. Prace. Mat. 46, 113-129 (2006)

33. Jiménez-Melado, A.; Llorens-Fuster, E.: A renorming of $\ell_{2}$, rare but with the fixed-point property. Int. J. Math. Math. Sci. 2003(65), 4115-4129 (2003) 
34. Jiménez-Melado, A.; Llorens-Fuster, E.: A class of renormings of $\ell_{2}$ with the fixed point property. J. Nonlinear Convex Anal. (in press)

35. Jiménez-Melado, A; Llorens-Fuster, E.; Saejung, S.: The von Newman-Jordan constant, weak orthogonality and normal structure in Banach spaces. Proc. Am. Math. Soc. 134, 355-364 (2006)

36. Karlovitz, L.A.: Existence of a fixed point for a nonexpansive map in a space without normal structure, Pac. J. Math. 66, $153-159$ (1976)

37. Khamsi, M.A.: Étude de la propriété du point fixe dans les espaces de Banach et les espaces métriques, Thèse de Doctorat de l'Université Paris VI (1987)

38. Khamsi, M.A.; Kirk, W.A.: An Introduction To Metric Spaces and Fixed Point Theory Pure and Applied Mathematics. Wiley and Sons, N.Y. (2001)

39. Kirk, W.A.: A fixed point theorem for mappings which do not increase distances. Am. Math. Monthly 72, 1004-1006 (1965)

40. Kirk, W. A.: Some questions in metric fixed point theory. Recent Advances on Metric Fixed Point Theory (Seville, 1995), vol. 48, pp. 73-97, Ciencias, Univ. Sevilla, Seville (1996)

41. Kutzarova, D.; Prus, S.; Sims, B.: Remarks on orthogonal convexity of Banach spaces. Houston J. Math. 19, 603-614 (1993)

42. Kirk. W.A.; Sims, B. (eds.) Handbook of Metric Fixed Point Theory. Kluwer Academis Publishers, Dordrecht (2001)

43. Lifshitz, E. A.: Fixed point theorems for operators in strongly convex spaces. Voronez Gos. Univ. Trudy Mat. Fak. 16, 23-28 (1975) (in Russian)

44. Lim, T.C.: Asymptotic centers and nonexpansive mappings in conjugate Banach spaces. Pac. J. Math. 90, 135-143 (1980)

45. Lin, P.K.: Unconditional bases and fixed points of nonexpansive mappings. Pac. J. Math. 116, 69-76 (1985)

46. Llorens-Fuster, E.: Semigroups of mappings with rigid Lipschitz constant. Proc. Am. Math. Soc. 130, 1407-1412 (2002)

47. Llorens-Fuster, E.; Muñiz-Perez, O.: Some relationships between sufficient conditions for the fixed point property (Submitted)

48. Naidu, S.V.R.; Sastry, K.P.R.: Convexity conditions in normed linear spaces. J. Reine Angew. Math. 297, 35-53 (1976)

49. Nelson, J.L.; Singh, K. L.; Whitfield, J.H.M.: Normal structures and nonexpansive mappings in Banach spaces. Nonlinear analysis, pp. 433-492. World Science Publishing, Singapore (1987)

50. Mazcuñán Navarro, E.: Geometry of Banach Spaces in Metric Fixed Point Theory. Ph. D. Thesis, Univ. of Valencia, Spain (2003)

51. Mazcuñán Navarro, E.: Stability of the fixed point property in Hilbert spaces. Proc. Am. Math. Soc. 134, 129-138 (2006)

52. Prus, S.: Banach spaces which are uniformly noncreasy. Nonlinear Anal. 30, 2317-2324 (1997)

53. Prus, S.; Szczepanik, M.: Nearly uniformly noncreasy Banach spaces. J. Math. Anal. Appl. 307, 255-273 (2005)

54. Saejung, S.: Convexity conditions and normal structure of Banach spaces. J. Math. Anal. Appl. 344, 851-856 (2008)

55. Sims, B.: Orthogonality and fixed points of nonexpansive maps. Proc. Center Austral. Math. Nat. Univ. 20, 179-186 (1988)

56. Sims, B.: A class of spaces with weak normal structure. Bull. Austral. Math. Soc. 49, 523-528 (1994)

57. Sims, B.; Smyth, M.: On non-uniform conditions giving weak normal structure. First International Conference in Abstract Algebra (Kruger Park, 1993). Quaestiones Math. 18(1-3), 9-19 (1995)

58. Sims, B.; Smyth, M.A.: On some Banach space properties sufficient for weak normal structure and their permanence properties. Trans. Am. Math. Soc 351, 497-513 (1999)

59. Smith, M.A.; Turret, B.: A reflexive LUR Banach space which laks normal structure. Can. Math. Bull. 28, $492-494$ (1985)

60. Sullivan, F.: A generalization of uniformly rotund Banach spaces. Can. J. Math. 31, 628-636 (1979)

61. Tan, K.-K.; Xu, H.K.: On fixed point theorems of nonexpansive mappings in product spaces. Proc. Am. Math. Soc. 113(4), 983-989 (1991)

62. Smyth, M.A.: Aspects of the Fixed Point Theory for some Metrically Defined Maps. Ph. D. dissertation, University of Newcastle, Australia (1994)

63. Turett, B.: A dual view of a theorem of Baillon. Nonlinear Analysis and Applications (St. Johns, Nfld., 1981). Lecture Notes Pure and Applied Mathematics, vol. 80, pp. 279-286. Dekker, New York (1982)

64. van Dulst, D.: Some geometric properties related to normal structure. Nonlinear Analysis and Applications (St Johns, Nfld., 1981). Lecture Notes in Pure and Applied Mathematics, vol. 80, pp. 155-162. Dekker, New York (1982)

65. van Dulst, D.; Sims, B.: Fixed points of nonexpansive mappings and Chebyshev centers in Banach spaces with norms of type (KK). Banach Space Theory and its Applications (Bucharest, 1981). Lecture Notes in Mathematics, vol. 991. Springer, Berlin, pp. 35-43 (1983)

66. Wiśnicki, A.: Towards the fixed point property for superreflexive spaces, Bull. Austral. Math. Soc. 64, 435-444 (2001)

67. Zidler, V.: On some rotundity and smoothness properties of Banach spaces. Dissertationes Math. Rozprawy Mat. 87, 33 (1971) 